\title{
Unsuspected rupture in unscarred uterus at an early gestation: a clinical dilemma
}

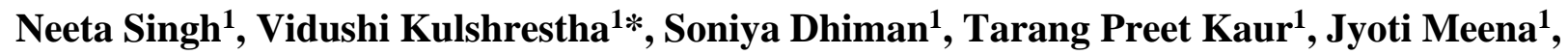 \\ Sandeep Mathur ${ }^{2}$
}

${ }^{1}$ Department of Obstetrics and Gynaecology, ${ }^{2}$ Department of Pathology, All India Institute of Medical Sciences (AIIMS), New Delhi, India

Received: 18 January 2022

Accepted: 08 February 2022

\section{*Correspondence:}

Dr. Vidushi Kulshrestha,

E-mail: drvidushi.kul@gmail.com

Copyright: ( ) the author(s), publisher and licensee Medip Academy. This is an open-access article distributed under the terms of the Creative Commons Attribution Non-Commercial License, which permits unrestricted non-commercial use, distribution, and reproduction in any medium, provided the original work is properly cited.

\begin{abstract}
Spontaneous uterine rupture in early second trimester is extremely rare specially in absence of predisposing factors. A pregnant patient presented at 15 weeks' gestation with lower abdominal pain and vomiting since two days. Ultrasound findings and deranged liver function tests reinforced the diagnosis of chronic liver disease with pregnancy. Clinical course a day later raised suspicion of ruptured uterus and patient was immediately taken for laparotomy. Intraoperatively, hemoperitoneum and $4 \mathrm{~cm}$ fundus rent was found. Total hysterectomy was performed and histopathology revealed placenta percreta as the probable cause of spontaneous rupture. The case highlights that a high index of clinical suspicion, an attentive daily clinical examination and immediate surgical management can avert life-threatening catastrophe.
\end{abstract}

Keywords: Early gestation, Placenta percreta spontaneous, Uterine rupture

\section{INTRODUCTION}

Uterine rupture is a potentially life-threatening obstetric complication which usually occurs peripartum or in scarred uterus. Uterine rupture in first or early second trimester in an unscarred uterus is extremely rare. The nonspecific symptoms of pain and vomiting may mimic symptoms of normal pregnancy and if pain is severe, medical or surgical emergency is suspected after ruling out an ectopic pregnancy. Here we report a rare case of rupture in an unscarred uterus at an early gestation of 15 weeks, in which clinical features of a medical disease as well as insidious onset of symptoms masqueraded the actual diagnosis. However, her sudden deterioration was timely detected and managed.

\section{CASE REPORT}

A 32-year-old, gravida-3 para-2, presented at $15^{+6}$ weeks of gestation to casualty with lower abdominal pain and vomiting since two days. She had two prior uncomplicated term vaginal deliveries with no significant previous history. This was an unplanned pregnancy diagnosed with urine pregnancy test at 2 months amenorrhoea. Patient had not received any antenatal care.

On admission patient was hemodynamically stable, fundal height corresponded to16 weeks with well-maintained uterine contour. Mild abdominal distention was noted with no tenderness. Transabdominal ultrasound revealed $15^{+5}$ weeks single live intrauterine fetus with fundo-anterior placenta, moderate intraperitoneal fluid and coarse echotexture of liver, suggesting liver disease. Laboratory analysis showed haemoglobin of $4.3 \mathrm{~g} / \mathrm{dL}$, TLC $7900 / \mathrm{mm}^{3}$, platelet $63000 / \mathrm{mm}^{3}$, PT-INR of 1.1 and deranged serum liver function tests (bilirubin $2.05 \mathrm{mg} / \mathrm{dL}$, aspartate-transferase $90 \mathrm{IU} / \mathrm{L}$, alanine-transferase $78 \mathrm{IU} / \mathrm{L}$, alkaline-phosphatase 401 IU/L). Hence initial clinical impression was intrauterine pregnancy with chronic liver disease (CLD) with moderate ascites with severe anaemia. 
Patient's vomiting subsided on antiemetics. Two units of packed red cells (PRBC) were transfused. While patient was being evaluated for CLD, a day later, she developed sudden onset abdominal pain followed by suddenly deteriorating hemodynamic parameters (pulse rate $124 /$ minute, blood pressure $80 / 50 \mathrm{mmHg}$ ). On palpation, abdomen was tender and uterine contour could not be defined. Henceforth an immediate transvaginal scan was done which showed empty uterine cavity. There was discontinuity of uterine contour at fundus with free fluid (Figure 1). Provisional diagnosis of ruptured uterus was considered and emergency laparotomy was planned after taking informed consent for hysterectomy also.

Intraoperatively, there was 2.5 litres of hemoperitoneum. Fetus was lying entangled in the omentum. Bilateral tubes and ovaries were healthy. A $4 \mathrm{~cm}$ fundal rent towards left cornua was noted which was profusely bleeding. On exteriorization, placental bits were found attached at rupture site with friable thin margins and increased vascularity (Figure $2 \mathrm{~A}$ and $\mathrm{B}$ ). Considering the appearance of uterus unsalvageable, total hysterectomy was undertaken instead of repairing the defect. The patient received four units packed red cells, two fresh frozen plasma and two random donor platelets. Patient had an uneventful recovery and was discharged on $7^{\text {th }}$ postoperative day.

The histopathology revealed chorionic villi invading through the entire myometrium up to serosa with areas of necrosis and haemorrhagic infarction, consistent with placenta percreta (Figure $3 \mathrm{~A}$ and $\mathrm{B}$ ).

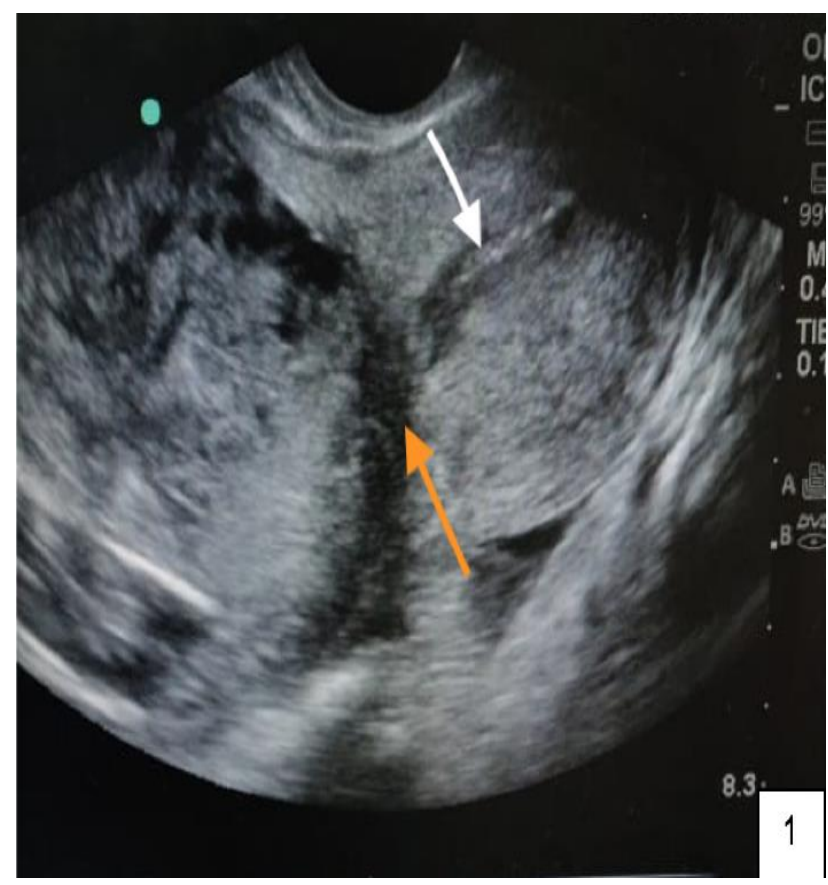

Figure 1: Transvaginal ultrasound showing empty uterine cavity (white arrow); discontinuity at upper segment (yellow arrow) raising suspicion of uterine rupture.

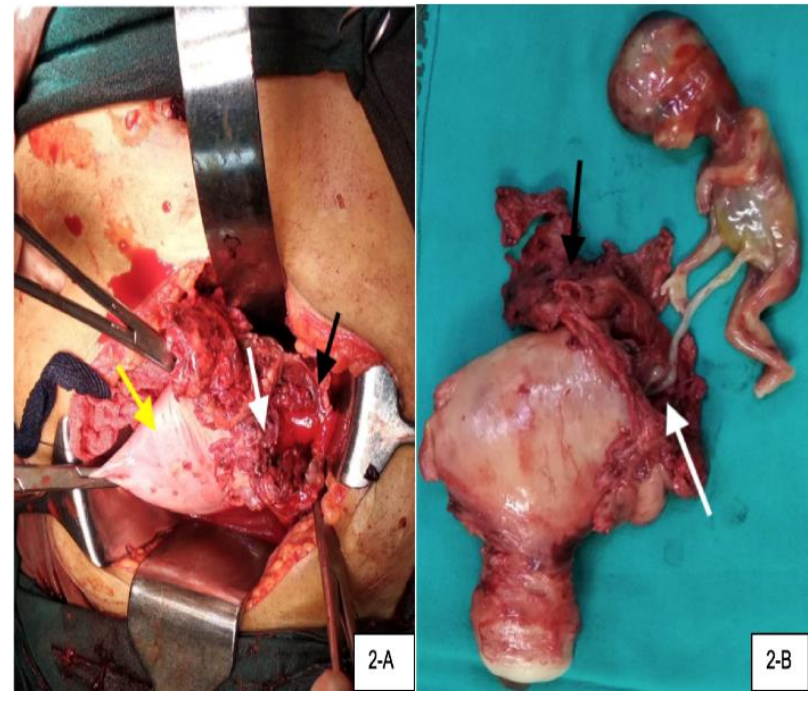

Figure 2 (A and B): Intraoperative findings and specimen; showing rupture near uterine insertion of left fallopian tube (black arrow), placental bits attached to ruptured site (white arrow) of uterus (yellow arrow).

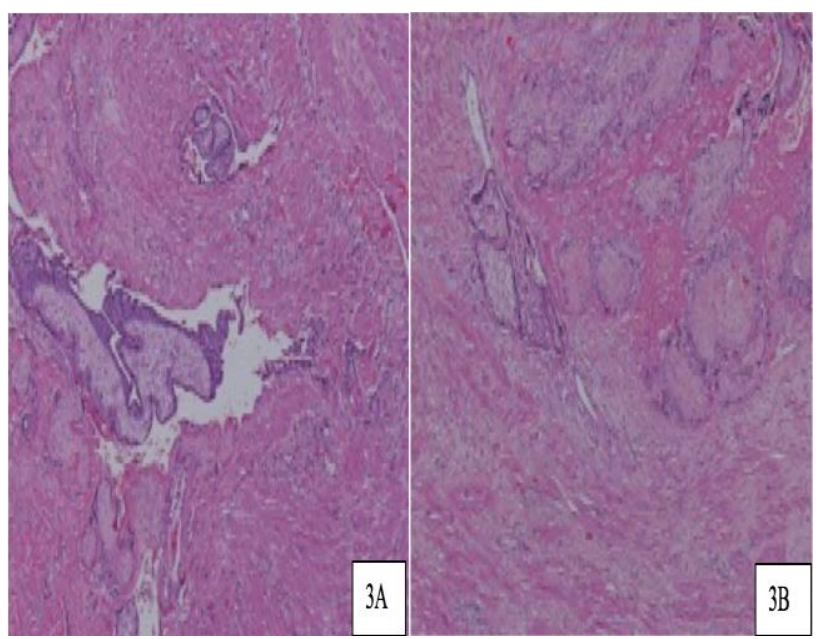

Figure 3 (A and B): Histopathology showing placenta percreta at the site of spontaneous rupture.

\section{DISCUSSION}

On admission, the reported patient was hemodynamically stable with viable intrauterine pregnancy-and fundal placenta. She had an unscarred uterus with no predisposing risk factors. Her clinical presentation of abdominal pain, vomiting; with heterogenous liver echotexture and ascites on ultrasound; with raised bilirubin and deranged transaminases; favoured the diagnosis of pregnancy with chronic liver disease thus masquerading the insidious rupture.

Spontaneous uterine rupture is a rare pregnancy complication which usually occurs in third trimester or during labor and mostly in a scarred uterus with prior caesarean or manual removal of placenta, myomectomy, 
septal resection, metroplasty or instrumentation such as dilatation and curettage. ${ }^{1}$ Other peripartum risk factors are obstetric manoeuvres, malpresentations, uterine hyperstimulation, curettage, injudicious oxytocin use or uterine diverticula. ${ }^{2}$ Scarred uterus also predisposes to development of placenta accreta syndrome (PAS) which is an independent risk factor for uterine rupture in early gestation also. ${ }^{3}$

Uterine rupture is even rarer in first or second trimesters in an unscarred uterus. Reported incidence of unscarred uterine rupture (UUR) is 1 in 17,000-20,000 pregnancies. $^{4}$ Associated risk factors for UUR are multiparity ( $\geq 4$ births) with short pregnancy intervals, advanced maternal age, multiple pregnancies, previous endometritis and misoprostol use. ${ }^{1}$ Though rare but PAS associated rupture in early pregnancy, as occurred in our patient, is also reported in unscarred uterus due to pathological invasion of the placenta through uterine wall. ${ }^{5,6}$

Abdominal pain with vomiting in early pregnancy can be a minor ailment of pregnancy or it may be due to medical, surgical or gynaecological emergencies including liver disorders, appendicitis, diverticulitis, peritonitis, urinary tract infection, ovarian torsion, haemorrhagic corpus luteum and heterotopic pregnancy. ${ }^{7}$ Hence, acute abdomen is nonspecific and has to be carefully evaluated to make a definitive diagnosis.

Free fluid in peritoneal cavity on ultrasound is also a nonspecific finding which may be seen in ascites, bowel perforation, ruptured aortic aneurysm; though ectopic pregnancy remains the most important differential in early pregnancy. Diagnosis of ectopic pregnancy should always be considered when a pregnant patient presents with acute abdomen in early pregnancy with free fluid, which in our case was excluded due to documented viable intrauterine pregnancy. Lee et al recently reported eleven published cases of uterine rupture between 13 and 21 weeks and suggested suspecting rupture if ultrasound shows intraperitoneal fluid with gravid uterus. ${ }^{6}$

Our case had a documented intrauterine pregnancy with well-maintained uterine contour and in absence of any abdominal tenderness, deteriorating vitals, or signs of shock, any obstetrical emergency seemed unlikely. Besides coarse liver echotexture with ascites with deranged liver function tests suggested liver pathology.

Clinching a diagnosis of UUR needs a high index of suspicion especially in early gestation. Free fluid in our case was thought to be ascites secondary to the liver disease. Surgical causes were not suspected as patient was hemodynamically stable. In a similar case report, ruptured uterus was masked due to co-existing gastroenterological symptoms at 26 weeks gestation. ${ }^{8}$

The site of rupture in early pregnancy is usually fundus, in contrast to lower uterine segment in third trimester, hence vaginal bleeding may be absent due to collection in peritoneal cavity, delaying the diagnosis. ${ }^{9}$ Even in cases with previous caesarean, scar may be spared in early pregnancy with rupture occurring at the fundus. ${ }^{3}$

Mostly all reported patients require laparotomy, though diagnostic laparoscopy can help in confirming the diagnosis and can be attempted in stable patient if in dilemma. Treatment is to be individualized based on the size of defect, parity, age and patient's hemodynamic status; with uterine repair or hysterectomy as the options. Repair is usually combined with bilateral tubal ligation to avoid future risk of rupture. Surgical repair of spontaneous rupture at 21 weeks followed by pregnancy progression to live birth is also reported. ${ }^{10}$ Laparoscopic approach may be favoured in haemodynamically stable patients when avoiding uterine manipulator, intrauterine vasopressin, lateralizing ureters and retroperitoneal dissection to secure uterine arteries at the origin may increase safety margin. ${ }^{6}$ In our case, hysterectomy was performed as tissues at the defect were very friable to hold any suture and patient had two live children.

Histopathology in our case revealed placenta percreta, which was the probable cause of rupture even though the uterus was not scarred. Only few cases are published describing placenta percreta as a reason for spontaneous rupture before 21 weeks' gestation; but most of these had scarred uterus with history of caesarean or curettage. ${ }^{11}$

The sensitivity of ultrasound for diagnosing placenta accreta in first and second trimesters is only $41 \%$ and $60 \%$ respectively. $^{12} \mathrm{~A}$ case-report has highlighted that ultrasound documentation of intrauterine pregnancy does not exclude uterine rupture in presence of free fluid in peritoneal cavity. ${ }^{14}$ Hence without a high risk of suspicion, diagnosis and appropriate management of uterine rupture can be delayed. In retrospect, it is possible that in our case, blood was slowly trickling from placental site leading to hemoperitoneum. At the event of actual rupture, patient collapsed. Ambrogi et al similarly described an insidious onset uterine rupture at 13 weeks due to placenta percreta in a woman who first presented at 9 weeks and then at 13 weeks with moderate abdominal pain with transvaginal ultrasound showing intrauterine pregnancy with even textured myometrium and moderate fluid in abdominal cavity including hepato-renal pouch. She was being managed expectantly when she suddenly went into hypovolemic shock and rupture was recognized on videolaparoscopy. ${ }^{3}$

The index case highlights the importance of daily clinical evaluation. Loss of uterine contour which was well documented only a day earlier and new onset abdominal tenderness clinched the suspicion of uterine rupture which was confirmed immediately on ultrasound.

\section{CONCLUSION}

Spontaneous uterine rupture in early second trimester of pregnancy is very rare especially in absence of identifiable 
risk factors. It should be kept as one of the differentials of abdominal pain during pregnancy irrespective of gestation. The current case highlights that a high index of clinical suspicion, an attentive clinical examination and immediate surgical management can avert life-threatening catastrophe.

\section{ACKNOWLEDGEMENTS}

Author would like to thanks to all authors contributed in the patient management and follow-up.

Funding: No funding sources Conflict of interest: None declared

Ethical approval: Not required

\section{REFERENCES}

1. Abbas AM, Hussein RS, Ali MN, Shahat MA, Mahmoud AR. Spontaneous first trimester posterior uterine rupture in a multiparous woman with scarred uterus: A case report. Middle East Fertil Soc J. 2018;23:81-83.

2. Uzun I, Yıldırım A, Kalelioglu I, Has R. Spontaneous rupture of unscarred uterus at 27 weeks of gestation. Arch Gynecol Obstet. 2010;281:999-1001.

3. Ambrogi G, Ambrogi G, Marchi AA. Placenta Percreta and uterine rupture in the first trimester of pregnancy. Case Rep. Obstet Gynecol. 2018;2018:6842892.

4. Ofir K, Sheiner E, Levy A, Katz M, Mazor M. Uterine rupture: differences between a scarred and an unscarred uterus. Am J Obstet Gynecol. 2004;191(2):425-9.

5. LeMaire WJ, Louisy C, Dalessandri K, Muschenheim F. Placenta percreta with spontaneous rupture of an unscarred uterus in the second trimester. Obstet Gynecol. 2001;98(5):927-9.

6. Lee F, Zahn K, Knittel AK, Morse J, Louie M. Laparoscopic hysterectomy to manage uterine rupture due to placenta percreta in the first trimester: A case report. Case Rep Womens Health. 2020;25:e00165.

7. Ijaz S, Mahendru A, Sanderson D. Spontaneous uterine rupture during the 1st trimester: a rare but lifethreatening emergency. J Obstet Gynaecol. 2011;31(8):772.

8. Kashyap P, Prasad S, Singh CB. A Rare Case of Second Trimester Uterine Rupture in Unscarred Uterus. Int J Med Res Health Sc. 2017;6(11):105-7.

9. Vaknin Z, Maymon R, Mendlovic S, Barel O, Herman A, Sherman D. Clinical, sonographic, and epidemiologic features of second-and early thirdtrimester spontaneous antepartum uterine rupture: a cohort study. Prenat Diagn. 2008;28:478-84.

10. Hawkins L, Robertson D, Frecker H, Berger H, Satkunaratnam A. Spontaneous uterine rupture and surgical repair at 21 weeks gestation with progression to live birth: a case report. BMC Pregnancy Childbirth. 2018;18:132.

11. Cecchini F. First Trimester Uterine Rupture: A Case Report and Literature Review. Int J Environ Res Public Health. 2020;17(8):2976

12. Rahimi-Sharbaf F, Jamal A, Mesdaghinia E, Abedzadeh-Kalahroudi M, Niroomanesh S, and Atoof F. Ultrasound detection of placenta accreta in the first trimester of pregnancy. Iran J Reprod Med.2014;12:421-6.

13. Acton C, King V, Whitehead J. Sonographic diagnosis of uterine rupture with a successful outcome. Aust N Z J Obstet Gynaecol. 2004;44:4734.

Cite this article as: Singh N, Kulshrestha V, Dhiman S, Kaur TP, Meena J, Mathur S. Unsuspected rupture in unscarred uterus at an early gestation: a clinical dilemma. Int J Reprod Contracept Obstet Gynecol 2022;11:969-72. 